\title{
Seroprevalence of Syphilis Infection among Apparently Clinically Healthy Blood Donors in Northern Mid - Karnataka
}

\author{
Bhakti V. Kulkarni, Basavaraj P. Bommanahalli* and Rajshekhar T. Pawadshettar \\ Dept of Pathology, Gadag Institute of Medical Sciences, Karnataka, India
}

\begin{abstract}
Background: Blood transfusion is one of the important modalities of treatment in hospitals these days. Transfusion transmissible infections(TTI) are one of the important complications of blood transfusion.

Objective: To study the seroprevalence of syphilis infection among healthy blood donors in our region

Materials and Methods: This is a retrospective study done over a period of 3 years from August 2015 to July 2018 in two blood banks of Gadag i.e. Gadag Institute of Medical Sciences (GIMS) blood bank and Indian Medical Association (IMA) blood bank. A total of 20,144 blood donors were included in the study. It included both voluntary donors and replacement donors. Donors were screened using donor questionnaire form followed by general physical examination for medical and surgical illness. Eligible blood donors donated blood and their blood samples were subjected to a test that detected the presence of non treponemal antibodies by using rapid plasma reagin (RPR) test kit.

Result: In the present study, the total number of donors is 20,144. Out of this, 19,676 (97.67\%) were males and $468(2.33 \%)$ were females. Total number of seropositive cases of syphilis was 5 . The overall seroprevalence was $0.023 \%$. All the seropositive cases were males. The prevalence of seropositive cases among voluntary donors was $0 \%$ and among replacement donors was $0.14 \%$
\end{abstract}

Conclusion: Seroprevalence of syphilis in the region of Gadagis $0.023 \%$

\section{Keywords: Seroprevalence, Replacement Donors, Voluntary Donors}

\section{Introduction}

Timely intervention is very essential in treating any patient. Blood transfusion serves this best. At the same time, unsafe transfusion practices puts many patients at risk of acquiring many complications. One of the significant complications of blood transfusion is transfusion transmissible infections (TTI) like Hepatitis B, Hepatitis C, Retroviral disease, Syphilis and Malaria. It is essential to curb the transmission of syphilis. The study on seroprevalence helps to identify the magnitude of the problem and also in identifying the various risk groups. ${ }^{1}$

Syphilis is one of the health burdens in the world. It is caused by a bacterium Treponema pallidum. It is transmitted by direct contact with chancre, sexually, vertically from mother to foetus and through transfusion of contaminated blood. The course of the disease can be divided into three stages: Primary, secondary and tertiary.

Different stages of the treponematoses require different testing modalities. In the early stages when lesions are present, highly specific microscopic techniques like dark field microscopy and fluorescence microscopy can be used. In the later stages, a variety of serological tests are required.
The serological tests are comprised of non treponemal tests and treponemal tests.

The nontreponemal assays detect antibodies to lipoprotein material and the cardiolipin released from the cells damaged by treponemes. Hence, these tests are not specific for treponema. Some of the non treponemal tests are veneral disease research laboratory (VDRL), rapid plasma reagin (RPR), unheated serum reagin (USR) and others. ${ }^{2}$

RPR is macroscopic, non treponemal, flocculation card test which is used as a screening test. The RPR test uses a stabilised suspension of antigen. Charcoal particles are added to it to improve the visualisation of the test reaction. It is a simplified version of VDRL test. ${ }^{3}$ The non treponemal tests detect IgG and IgM antibodies as early as 6 days post infection. ${ }^{4}$

Few of treponemal tests are Treponema pallidum hemagglutination test (TPHA), Treponema pallidum particle agglutination assay (TPPA), fluorescent treponemal antibody absorption assay (FTA-ABS) and Enzyme linked immunosorbent assay (ELISA). The TPPA test is superior to TPHA test while FTA-ABS is the first serological test to become reactive during the primary stage of disease. ${ }^{4}$ 


\section{Objective}

To study the seroprevalence of syphilis infection among healthy blood donors in our region

\section{Materials and Methods}

This is a retrospective study done over a period of 3 years from August 2015 to July 2018 in two blood banks attached to Gadag Institute of Medical Sciences (GIMS) and Indian Medical Association (IMA). A total of 20,144 blood donors were included in the study. It included both replacement donors and voluntary donors. Family members, relatives or friends of the patients were grouped as replacement donors. People who donated either in blood bank or blood camps voluntarily without any favour in return and any pressure were grouped as voluntary donors. Donors were given a questionnaire form that comprised of donor register form which included donor's name, gender, address, occupation, date of previous donation, pregnancy status and risk factors such as surgery, hospitalisation, hypertension, diabetes, blood transfusion. General physical examination and haemoglobin screening was performed.

Inclusion criteria: Clinically healthy individuals between 18 to 65 years of age with the body weight of above $45 \mathrm{~kg}$ and haemoglobin $>12.5 \mathrm{~g} / \mathrm{dl}$, with no apparent medical or surgical illness, were included in the study.

Exclusion criteria: Pregnant women, patients with sexually transmitted diseases, drug abusers, sex workers were excluded from blood donation. Individuals having chronic diseases, cardiac diseases are permanently deferred. Patients with anaemia, low body weight were temporarily deferred.

After blood donation, the samples were obtained for serological testing. Donor samples were processed for the detection of non treponemal antibodies by using rapid plasma reagin (RPR) test kit, as per the procedure given by the manufacturer. Patient's serum was placed on the circle of test card. To this the antigen suspension was added and the suspension was mixed well. After about 4-8 minutes, we observed for flocculation, seen as clumping on the card which was taken as positive test and if there was no clumping then it was taken as negative test.

Statistics: Data was entered in Microsoft Excel sheet and the seroprevalence was calculated with respect to age, gender and type of donor. The statistical significance was determined by calculating $\mathrm{p}$ value using chi-square test.

\section{Results}

The overall seroprevalence of syphilis cases was $0.023 \%$. Seroprevalence was higher among replacement donors when compared to voluntary donors, accounting for about $0.14 \%$, which was statistically significant ( $\mathrm{p}$ value $<0.001$ ). Prevalence was high among male donors accounting for about $0.025 \%$ and in females it was $0 \%$. The difference of seroprevalence among gender was statistically insignificant ( $\mathrm{p}$ value $>0.7302$ ). The seroprevalence was high in the age group 26-30 years accounting for $0.056 \%$ (Table 1). Seroprevalence of syphilis among donors showed a decreasing trend from 2015 to 2018.(Graph 1).

\section{Discussion}

It is estimated that there is $1 \%$ chance of transfusion associated problems including TTI for every unit of blood. ${ }^{5}$

In the present study, the overall seroprevalence of syphilis infection among blood donors is $0.023 \%$ which is in concordance with a study done by Garg et $\mathrm{al}^{6}$ (Table 2).

In our study, the seroprevalence of syphilis is highest among males accounting for $0.025 \%$ and none of the female donors turned out be seropositive for syphilis. The difference was statistically insignificant ( $p$ value $>0.7302$ ). The reason for higher seroprevalence among males is due to minimum participation by females due to cultural norms and prevalence of anemia among them. In a study done by Chaudhary et al, the seroprevalence of syphilis among male donors was $0.16 \%$ and that in female donors was $0.07 \% .^{13}$

In the present study, the seroprevalence of syphilis infection is high in the age group 26-30 years accounting for $0.56 \%$. This is in concordance with a study done by Khagesan et al where the maximum seropositive donors were in the age

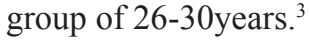

The present study reveals that the seroprevalence of syphilis is highest among replacement donors accounting for $0.14 \%$ while none of the voluntary donors showed seroreactivity for syphilis. The difference between seroprevalence between voluntary and replacement donors is statistically significant ( $\mathrm{p}$ value $<0.001 \%$ ). A study done by Garg et al ${ }^{6}$ showed highest seroprevalence among replacement donors accounting for $0.239 \%$. Voluntary donors donate blood of their own free will and with no any medical risk factors. Hence, the prevalence of syphilis is much lower when compared to replacement donors.

The seroprevalence of syphilis among the blood donors showed a decreasing trend from August 2015 to July 2018. In a study done by Singh et al, there was a decreasing trend of seroprevalence of the syphilis infection among blood donors from the year 2000 to $2002 .{ }^{9}$

Syphilis infection can increase the susceptibility to HIV infection. At the same time, HIV can alter the clinical 
course of syphilis, increase the likelihood of relapse and confound the diagnosis of neurosyphilis. ${ }^{12}$ Non treponemal test titres may be higher among HIV-positive patients than among HIV negative patients. ${ }^{14}$

Table 1: Seroprevalence of Syphilis among blood donors by demographic characteristics.

\begin{tabular}{|c|c|c|c|}
\hline Variable & No. of Donors & Seropositive cases & Seroprevalence (\%) \\
\hline Type & & 0 & 0 \\
\hline Voluntary & 16541 & 5 & 0.14 \\
\hline Replacement & 3603 & 5 & 0.025 \\
\hline Gender & 19676 & 0 & 0 \\
\hline Male & 468 & & 0.013 \\
\hline Female & & 1 & 0.056 \\
\hline Age & 7753 & 3 & 0.03 \\
\hline $26-30$ & 5283 & 1 & 0 \\
\hline $31-35$ & 3287 & 0 & 0 \\
\hline $36-40$ & 1788 & 0 & 0 \\
\hline $41-45$ & 1088 & 0 & 0 \\
\hline $46-50$ & 523 & 0 & $\mathbf{0 . 0 2 3}$ \\
\hline$>50$ & 422 & $\mathbf{5}$ & \\
\hline Total & $\mathbf{2 0 1 4 4}$ & & \\
\hline
\end{tabular}

Table 2. Comparison of seroprevalence of Syphilis among blood donors in different studies.

\begin{tabular}{|c|c|c|c|}
\hline Authors & Voluntary donors(\%) & Replacement donors(\%) & Total (\%) \\
\hline Srikrishna et al (1991) & - & - & 1.6 \\
\hline Garg et al (1998) & 0.129 & 0.239 & 0.22 \\
\hline Sonawane et al (2003) & 0.33 & 1.12 & 0.87 \\
\hline Singh et al (2005) & 1.4 & 2.8 & 2.6 \\
\hline Bhattacharya (2007) & - & - & 0.8 \\
\hline Arora et al (2010) & - & - & 0.9 \\
\hline Pallavi et al (2011) & - & 0.05 & 0.28 \\
\hline Khageshan et al (2016) & 0 & 0.14 & 0.04 \\
\hline Present study & 0 & & 0.023 \\
\hline
\end{tabular}

Graph 1. Yearwise trend of seroprevalence of syphilis among blood donors Descriptive

\begin{tabular}{|c|c|c|c|}
\hline \multicolumn{1}{|c|}{ Year } & N & Mean & Std. Deviation \\
\hline Aug15-July16 & 171 & 1.98 & 0.152 \\
\hline Aug16-July17 & 170 & 2.00 & 0.000 \\
\hline Aug17-July18 & 96 & 1.99 & 0.102 \\
\hline Total & $\mathbf{4 3 7}$ & $\mathbf{1 . 9 9}$ & $\mathbf{0 . 1 0 6}$ \\
\hline
\end{tabular}

\begin{tabular}{|c|c|c|c|c|c|}
\hline \multicolumn{7}{|c|}{ ANOVA } & Fean Square & Sig. \\
\hline Syphilis & Sum of Squares & df & 0.023 & 2.073 & 0.127 \\
\hline Between Groups & 0.047 & 2 & 0.011 & & \\
\hline Within Groups & 4.896 & 434 & & & \\
\hline Total & $\mathbf{4 . 9 4 3}$ & $\mathbf{4 3 6}$ & & \\
\hline
\end{tabular}

$\mathrm{P}$ value is $>0.05$ not significant 


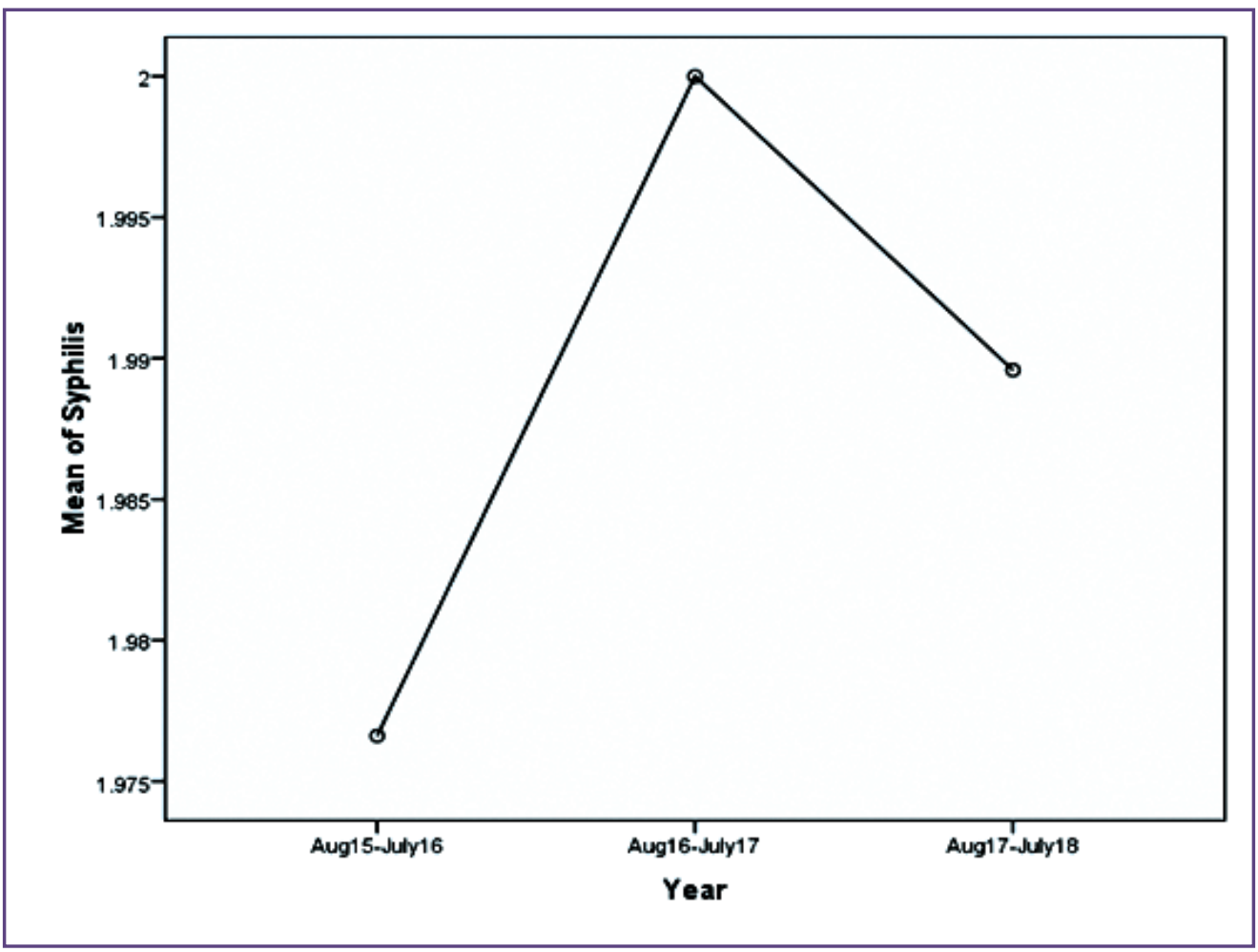

Trend Plot

\section{Conclusion}

We conclude that the seroprevalence of syphilis infection among blood donors in our region is low. The seroprevalence is high among economically productive age group and replacement donors. Hence, there is a need to encourage extensive donor blood screening, awareness among people regarding the spread of infection, achieve $100 \%$ non- renumerated voluntary donation and ensuring blood safety measures.

\section{References}

1. Bagiyalakshmi V, Gopal R, Elangovan RS. Seroprevalence of hepatitis B virus infection among volunatary blood donors at a tertiary care hospital blood bank-Tiruchirapalli. Int J Sci Stud. 2017;4(10):105-8

2. Mcpherson RA, Pincus MR (Eds). Henry's Clinical Diagnosis and Management by Laboratory Methods. 22nd edition. New Delhi: Reed Elsevier India Private Limited; 2012

3. Khageshan AP, Kulkarni KR, Baragundi MC. Seroreactivity of syphilis among blood donors of a blood bank. Annals of Pathology and Laboratory Medicine. 2016;3(1):41-4

4. Morshed MG, Singh AE. Recent trends in the serologic diagnosis of Syphilis. Clinical and Vaccine Immunology. 2015;22(2): 137-47
5. Arora D, Arora B, Kheterpal A. Seroprevlence of HIV, HCV and syphilis in blood donors in Southern Haryana. Indian J Pathol Microbiol. 2010;53(2):308-9

6. Garg S, Mathur DR, Garg DK. Comparison of seropositivity of HIV, HBV, HCV and syphilis in replacement and voluntary blood donors in western India. Indian $\mathrm{J}$ Pathol Microbiol. 2001;44(4):409-12

7. Srikrishna A, Sitalakshmi S, Damodhar P. How safe are our safe donors? Indian J Pathol Microbiol. 1999;42:411-6

8. Sonawane BR, Birare SD, Kulkarni PV. Prevalence of seroreactivity among blood donors in rural population. Indian J Med Sci. 2003;57:405-7

9. Singh B, Verma M, Kotru M, Verma K, Batra M. Prevalence of HIV and VDRL seropositivity in blood donors of Delhi. Indian J Med Res. 2005;122:234-6

10. Bhattacharya P, Chandra PK, Datta S, Banerjee Arup, Chakraborty S, Rajendran K, et al. Significant increase in $\mathrm{HBV}, \mathrm{HCV}, \mathrm{HIV}$ and syphilis infections among blood donors in West Bengal, Eastern India 2004-2005: Exploratory screening reveals high frequency of occult HBV infection. World J Gastroenterol. 2007;13(27):3730-3

11. Pallavi $\mathrm{P}$, Ganesh CK, Jayashree K, Manjunath CV. Seroprevalence and trends in transfusion transmitted infections among blood donors in a University hospital blood bank: A 5 year study. Indian J Hematol Blood Transfus. $2011 ; 27(1): 1-6$ 
12. Kaur G, Basu S, Kaur R, Kaur P, Garg S. Patterns of infections among blood donors in a tertiary care center: A retrospective study. Natl Med J India . 2010;23(3):147-9

13. Chaudhary V, Agarwal VK, Sexena KS, Upadhyay D, Singh A, Singh SP. Seroprevalence of common transfusion transmissible infections among blood donors in Western
Uttar Pradesh, India. Int J Med Sci Public Health. 2014;3(11):1381-4

14. Rompolo AM, Cannon RO, Quinn TC, Hook EW. Association of biologic false-positive reactions for syphilis with human immunodefiency virus infection. J Infect Dis. 1992;165(6):1124-6

*Corresponding author:

Dr. Basavaraj P. Bommanahalli, Professor and Head, Department of Pathology, Gadag Institute of Medical Sciences, Gadag, State: Karnataka, India;

Phone: +9199160 88221

Email: basupath@rediffmail.com

Financial or other Competing Interests: None. 